\title{
Metodologías participativas y patrimonio cultural inmaterial en los barrios
}

\author{
Participatory methods and intangible cultural \\ heritage in the neighbourhoods
}

\author{
Nuria Nebot-Gómez de Salazar \\ Universidad de Málaga \\ nurianebot@uma.es \\ https://orcid.org/0000-0001-6187-0972 \\ Eva Morales-Soler \\ Universidad de Málaga \\ evamorsol@uma.es \\ https://orcid.org/0000-0002-4182-9443 \\ Carlos Rosa-Jiménez \\ Universidad de Málaga \\ cjrosa@uma.es \\ https://orcid.org/000-0001-6356-8734
}

\begin{abstract}
Resumen
La protección del Patrimonio Cultural Inmaterial (PCI) resulta fundamental como herramienta de cohesión social en las comunidades locales, al infundir en ellas un sentimiento de identidad y continuidad, y promover el respeto a la diversidad y creatividad humana. Sin embargo, la salvaguardia de este tipo de patrimonio es algo muy reciente y existe todavía una falta de consenso acerca de criterios comunes y metodologías para su protección. De ahí la importancia de diseñar y poner en práctica metodologías participativas que permitan crear espacios de participación en torno a la gestión del PCI en los barrios. Este artículo muestra los resultados de una experiencia real llevada a cabo en el barrio de la Fontanalla en Málaga (España). Se propone una serie de acciones metodológicas innovadoras y abiertas, basada en la participación e implicación de vecinos/as y entidades locales en el propio diseño del proceso participativo a desarrollar. Los resultados confirman la idoneidad de dicha metodología para crear espacios de participación. Las conclusiones recogen algunas particularidades de la gestión comunitaria del PCI, mecanismos utilizados, dificultades encontradas y su impacto en la gobernabilidad participativa del barrio malagueño.
\end{abstract}

\section{Palabras clave}

Patrimonio cultural inmaterial, participación comunitaria, comunidades locales, memoria colectiva, barrios, oficios, artesanía.

Forma sugerida de citar: Nebot-Gómez de Salazar, N., Morales-Soler, E., \& Rosa-Jiménez, C. (2020). Metodologías participativas y patrimonio cultural inmaterial en los barrios. Universitas, 33, pp. 83-102. 


\begin{abstract}
The protection of the Intangible Cultural Heritage (PCI) is essential as a tool for social cohesion in local communities, instilling in them a feeling of identity and continuity, and promoting respect for diversity and human creativity. However, the safeguarding of this type of heritage is something very recent and there is still a lack of consensus about the common criteria and methodologies for its protection. Hence the importance of designing and implementing participatory methodologies that specifically create spaces for participation around the management of the PCI in neighborhoods. This article shows results obtained from a real experience carried out in the neighbourhood of La Fontanalla (Málaga, Spain). A series of innovative and flexible methodological actions are proposed, based on the involvement of neighbours and local entities in the design of the participatory process. The results carried out confirm the suitability of this methodology to create spaces for participation. The conclusions include some peculiarities of community management of the PCI, mechanisms used, difficulties that have been found and their impact on participatory governance in the Malaga neighborhood.
\end{abstract}

\title{
Keywords
}

Intangible cultural heritage, community participation, local communities, collective memory, neighbourhoods, trades, crafts.

\section{Introducción y estado de la cuestión}

\section{Patrimonio Cultural Inmaterial y la necesidad de investigar en metodologías de protección}

El patrimonio cultural inmaterial, en adelante PCI, queda claramente definido en el texto elaborado por la Convención para su salvaguardia celebrada en París - si bien su entrada en vigor no se produce hasta 2006refiriéndose a "los usos, representaciones, expresiones, conocimientos y técnicas que las comunidades, grupos y, en algunos casos, los individuos reconocen como parte integrante de su patrimonio cultural" (UNESCO, 2003, p. 2). De este documento se desprende la importancia del patrimonio inmaterial como herramienta que infunde en las comunidades un sentimiento de identidad y continuidad promoviendo el respeto de la diversidad cultural y 
creatividad humana. Investigaciones recientes (Carofilis \& García, 2015) atribuyen al patrimonio cultural un rol cualitativo y específico en la formación de comunidades que pueden guiar transformaciones del entorno físico y economías hacia espacios de identidad.

La protección de este tipo de patrimonio es algo muy reciente que requiere, todavía, de un trabajo de investigación, consenso y gobernanza de administraciones e instituciones. Entre las principales tareas que establece la Convención, destaca la identificación del PCI a través de la elaboración de inventarios. Sin embargo, no especifica cómo han de hacerse, las metodologías a implementar, o criterios claros y concretos para su discriminación, (UNESCO Cataluña, 2011) quedando abiertos a la interpretación y adecuación de los estados.

Tal y como expresan Escalona-Hernández et al. (2017) las metodologías empleadas para la elaboración de inventarios se orientan al patrimonio cultural tangible, y el patrimonio inmaterial se aborda de forma más limitada. Otras investigaciones (Dumas, 2016) coinciden en que no se ha priorizado el trabajo para la conservación del patrimonio inmaterial en la manera adecuada. Resultan de especial interés algunas publicaciones recientes sobre experiencias previas llevada a cabo, sobre todo, en relación a la gestión del patrimonio cultural inmaterial (UNESCO Cataluña, 2011; Van der Hammen-Malo et al., 2015), en las que, a través de casos reales, se pueden conocer algunas de las dificultades encontradas en los procesos. Estas experiencias confirman la falta de metodologías consensuadas y la necesidad de investigar criterios comunes para abordar el estudio y gestión del PCI.

Las propias administraciones empiezan a tomar conciencia de la importancia de proteger este patrimonio, y de consensuar políticas y criterios de intervención. En España, ante la falta de criterios comunes y metodologías de intervención en las diferentes comunidades autónomas, se estableció un Plan Nacional de Patrimonio Inmaterial (Plan Nacional para la Salvaguardia del Patrimonio Cultural Inmaterial, 2011) como marco común de referencia. En éste, se caracteriza el patrimonio inmaterial como un patrimonio rememorado, transmitido de generación en generación, recreado, y vinculado a emociones y registros sensoriales. Asimismo, se definen unos ámbitos del patrimonio inmaterial que, sin diferir de los establecidos en la Convención de París, se ajustan con mayor precisión a la realidad española: 1) actividades productivas, 2) creencias y rituales, 3) tradición oral, 4) representacio- 
nes, 5) ámbito musical, 6) alimentación y cocina, y 7) formas de socialización (Carrión-Gutiez, 2015).

En la actualidad, son muy limitados los catálogos o registros de este patrimonio inmaterial en España. Cabría destacar el trabajo del Atlas sobre Patrimonio Inmaterial de Andalucía, del Instituto Andaluz del Patrimonio Histórico (Carrera, 2009). Desde éste, se ha realizado una labor importante en las tareas de identificación, catalogación y difusión del patrimonio inmaterial de la región andaluza. Sin embargo, existe, todavía, un campo de estudio que no ha sido explorado: la identificación y salvaguardia del patrimonio cultural inmaterial vinculado a los barrios y las comunidades locales.

Lo que desde esta investigación se plantea, es utilizar el patrimonio inmaterial como una herramienta para impulsar procesos de participación y una vida comunitaria activa en las barriadas. El objetivo último no es la elaboración de un catálogo en sí mismo, sino la creación de espacios de participación en torno al patrimonio inmaterial que incentiven comunidades cohesionadas, diversas y creativas.

\section{Implicación de las comunidades locales en la salvaguarda del PCI}

De la lectura del texto elaborado por la Convención, se desprende el importante papel de las comunidades locales en la salvaguardia del PCI. Son las comunidades locales las que avalan y alimentan las tradiciones, y garantizan su mantenimiento y continuidad en el tiempo (Frieri, 2014). Las recomendaciones de la Convención (Unesco, 2003) inciden en la necesidad de hacer partícipes a las comunidades, los grupos y los agentes sociales en la definición, la localización y el inventario de su patrimonio cultural inmaterial. Otras investigaciones posteriores inciden en la necesaria participación, negociación y consenso con los portadores de este patrimonio (Mariano et al., 2014).

El papel de las administraciones es muy importante frente a la necesidad de salvaguardar el patrimonio inmaterial, empezando por su identificación, registro y catalogación, así como su difusión y promoción. Sin embargo, los procesos de patrimonialización son complejos y, en muchas ocasiones, se producen conflictos entre las concepciones institucionales y dinámicas locales (Lacarrieu \& Laborde, 2018; Sánchez-Carretero \& Jiménez-Esquinas, 2016). Algunas investigaciones muestran la necesidad e idoneidad de inte- 
grar opiniones de expertos y científicos con el conocimiento local como medio para abordar esta complejidad (Craps \& Brugnach, 2015). Si se han de priorizar algunas de las medidas a adoptar por parte de las administraciones locales, se insiste en la necesidad de implicar a las comunidades y grupos en la salvaguardia del patrimonio inmaterial como agentes que mantienen, transmiten y pueden gestionar el patrimonio inmaterial de sus barrios de una forma activa.

Este artículo propone una serie de acciones metodológicas para hacer partícipes a las comunidades, grupos y agentes sociales a lo largo de todo un proceso participativo. El objetivo principal de dicho proceso es definir, localizar y dar valor a su patrimonio cultural. Desde el convencimiento profundo de la necesidad de involucrar a las comunidades locales en la gestión de su patrimonio cultural, se ha planteado su implicación, incluso, en las fases preparatorias de diseño y planificación de actuaciones a realizar, junto al acompañamiento de técnicos y académicos.

Este conjunto de acciones metodológicas, cuyas bases más generales se muestran a continuación, será testado en diferentes barriadas malagueñas para poder extraer unas conclusiones acerca de su validez como forma de actuación extrapolable a diferentes ámbitos. De forma concreta, la aportación de la investigación es contribuir en el conocimiento y comparación de experiencias metodológicas frente a la gestión del patrimonio intangible. En este artículo se hace una valoración de los resultados obtenidos en el proceso participativo llevado a cabo en el barrio de la Fontanalla en Málaga (España).

\section{El barrio de la Fontanalla como caso de estudio}

El Arrabal de Fontanalla en Málaga (España) es un antiguo barrio extramuros de la antigua ciudad árabe (Machuca Santa-Cruz, 1987). Se trataba de una zona industrial cuya actividad principal era la actividad artesanal de la cerámica, lo que justifica el afloramiento de multitud de hornos arqueológicos medievales en las diferentes excavaciones realizadas. Estos restos son una muestra del barrio artesanal que fue y que, en la actualidad, se quiere potenciar a través del trabajo de algunos talleres de artesanía del vidrío, de la cerámica y de otros oficios (PTVMalaga, 2019).

Junto a estos afloramientos - antiguos y actuales - de artesanía y oficios se pueden encontrar multitud de elementos con valor patrimonial, tan- 
gible e intangible: pinturas murales en las fachadas de algunas de sus viviendas, tradicionales tipologías residenciales de casa-patio, calles estrechas e irregulares propias de la antigua trama urbana de la ciudad musulmana, etcétera (Racero-Calvo, 2014). Desde el punto de vista social y urbano, nos encontramos con un barrio de gran complejidad, con una tasa elevada de desempleo entre sus habitantes y un alto porcentaje de inmigrantes (Sánchez Gómez et al., 2013). Se han identificado problemas de falta de convivencia dentro de una comunidad local conformada por un vecindario autóctono, con un fuerte sentimiento de arraigo, frente a otros colectivos que habitan más recientemente en el barrio, y que adolecen del mismo. Además, su estructura urbana, caracterizada por su abigarrado caserío, calles estrechas e irregulares, un alto número de solares vacíos y el fuerte impacto de medianerías en el espacio urbano, acentúa los problemas de segregación social existentes.

Frente a esta situación de desarraigo de una parte de la comunidad local, representada por un alto número de inmigrantes, existe un sector muy activo en la vida comunitaria del barrio y su patrimonio cultural. En este contexto de desequilibrio y división, el objetivo del proceso participativo es implicar al mayor número de agentes y personas que permitan una mayor cohesión de la comunidad, reforzar el sentido de identidad y pertenencia al barrio, y en el mejor de los casos, fomentar el respeto de los habitantes hacia su entorno físico. De esta manera, la metodología diseñada tendría una repercusión directa y muy positiva en la gobernabilidad participativa del barrio. El objetivo de este artículo es diseñar y poner en práctica una serie de acciones metodológicas que permita crear espacios de participación en torno a la gestión del patrimonio inmaterial en los barrios y sus comunidades locales. De esta forma, el PCI se convierte en el medio o herramienta capaz de activar procesos participativos.

\section{Metodología}

La metodología precisa de un equipo multidisciplinar de especialistas con experiencia en la gestión participativa en el campo del urbanismo y la educación social. Por otro lado, el estudio y gestión del patrimonio inmaterial requiere una formación muy específica, más aún, si se considera la falta de referentes metodológicos existentes previamente mencionados. 
Asimismo, se define un ámbito o escala de actuación: los barrios como áreas de oportunidad para acceder a las agrupaciones ciudadanas, conocer sus necesidades y problemáticas, y atender sus demandas. Con objeto de involucrar a la comunidad local, tanto en el propio diseño del proceso participativo como en la planificación de las acciones a realizar en torno al patrimonio inmaterial de La Fontanalla, se establecen tres fases:

\section{Fase 1. Construcción de un mapa de agentes intervinientes protagonistas del proceso y grupo de trabajo}

a. A nivel preparatorio se ha contactado con los informantes clave, estos son los representantes de la Asociación de Vecinos y Comerciantes del Arrabal Fontanalla, representante del Museo del Vidrio y Cristal de Málaga, representantes del colectivo de artesanos además de algunas personas del vecindario.

b. Con el apoyo de los informantes clave, se ha elaborado un mapa de agentes intervinientes o sociograma del barrio que ha permitido identificar aquellas personas o sectores de la población que viven e intervienen en el contexto de estudio. Los agentes intervinientes prioritarios son los/as vecinos/as, pero, además, se ha incluido a personas vinculadas con la barriada que forman parte del sector técnico, político y empresarial.

c. A partir de aquí se ha establecido la vía idónea para realizar la comunicación y difusión de las actividades participativas propuestas, así como el formato, la hora y el lugar donde éstas se han realizado.

\section{Fase 2. Elaboración por los especialistas de una propuesta técnica inicial abierta y genérica de posibles actuaciones en torno al patrimonio inmaterial como base de trabajo y discusión con la comunidad local}

Resulta fundamental la elaboración de una propuesta inicial de acciones metodológicas lo suficientemente genérica como para implementarse en distintos contextos e idiosincrasias. Dicha propuesta inicial incluye una serie de actuaciones que se describen a continuación (la elección de acciones a desarrollar ha sido decidida por parte de la comunidad local a partir de 
esta propuesta). En la siguiente relación se incluyen todas las acciones que al inicio del proceso participativo se han propuesto al grupo de vecinos/as participantes. De todas ellas, se ha ejecutado y finalizado la acción a) y se ha iniciado la acción b).

a. El paseo colectivo. Esta acción se refiere a los paseos colectivos con la ciudadanía, con la expresa invitación de informantes privilegiados que fomenten el conocimiento de la historia, realidad y modos de vida del barrio. Esta práctica ha de facilitar que las personas que participen contribuyan en la construcción del relato sobre la vida en el barrio.

b. La memoria del barrio. Con esta acción se plantea la recopilación de fotos antiguas que recojan la memoria del barrio abierto al vecindario. Esta recopilación se puede realizar en un taller específico con este objetivo, así como a partir de un espacio virtual que invite a la ciudadanía a subir sus propias fotos. Estas imágenes pueden acompañarse con 'pequeños relatos' que permitan ir componiendo 'historias de vida' y 'elementos de valor patrimonial' del barrio.

c. Taller Discusión. Se plantea un taller con grupo de discusión para la caracterización cualitativa de los elementos identificados. Con las imágenes y relatos recopilados se realizará un taller abierto a la ciudadanía que permita ordenar y construir una 'historia vivida y los elementos patrimoniales del barrio' de forma colaborativa. Se trata de caracterizar los elementos recopilados de forma cualitativa, ordenados a partir de una línea de tiempo.

d. La exposición pública. La acción de exposición propone una construcción gráfica de una línea temporal donde se ordene el material recopilado y seleccionado en función del tipo de elemento que sea, del sector de población que se identifique con el mismo, y del origen temporal o secuencial en el que se pueda ubicar. Con esta secuencia de imágenes se puede realizar una exposición abierta y pública para abrir el debate a todo el barrio y/o ciudad.

e. El taller final. A modo de conclusiones se plantea un encuentro final o tertulia, con el objetivo de abrir el debate y terminar de priorizar los elementos patrimoniales, tangibles e intangibles, que de forma colectiva se puedan identificar a lo largo de los talleres. 


\section{Fase 3. Elaboración por parte de agentes locales y vecinos/as de un programa de actuaciones a realizar a partir de la propuesta técnica inicial de acciones metodológicas}

A partir de la propuesta técnica inicial de actuaciones, y tras una serie de reuniones participativas y abiertas a toda la comunidad local, se ha consensuado el programa de acciones metodológicas a realizar, fechas, lugares y personas e instituciones implicadas en cada una de ellas. Ante la dificultad de involucrar a ciertos sectores de la población en la asistencia a los talleres participativos, se ha considerado necesario realizar actividades con distintos formatos y espacios de participación.

\section{Resultados}

A partir de los primeros resultados puede hacerse una valoración de los mecanismos empleados, su repercusión en la gobernabilidad de la comunidad local, así como valorar la capacidad del patrimonio inmaterial y su salvaguardia como activador de procesos participativos.

\section{Fase 1: Mapa de agentes locales, grupo de trabajo y planificación de actuaciones}

El sociograma de la Fontanalla ha sido una tarea sencilla de realizar por tratarse de una comunidad donde ya existe una asociación de vecinos/as y comerciantes formada por personas que viven o trabajan en el barrio, representantes de instituciones locales, incluso, pequeños empresarios del entorno.

Esta asociación es muy activa y facilita la relación de sus habitantes con algunas de las principales instituciones del barrio como son el Museo de Vidrio y Cristal de Málaga, el centro educativo IES Vicente Espinel, el taller de Artesanía del Vidrío Viarca, el colectivo de artesanos, o los pequeños comercios del barrio. Todos ellos forman parte de esa comunidad local cuya implicación en el proceso ha resultado fundamental. La conformación de este mapa de agentes intervinientes podrá ampliarse a lo largo del proceso, según se vayan desarrollando las sucesivas fases del mismo. Una vez construido el sociograma, se ha formado un grupo de trabajo con los algunos agentes del barrio y técnicos/académicos: presidenta de la Asociación 
de Vecinos y Comerciantes Arrabal Fontanalla, director del Museo de Vidrio y Cristal de Málaga, un artesano con taller en el barrio, varias personas del vecindario, especialistas e investigadores en la gestión de procesos participativos de la Universidad de Málaga. La formación de este grupo transversal ha permitido la confluencia no jerarquizada de conocimientos y prácticas científicas, locales y artesanales, tratándose de una cuestión fundamental en este tipo de procesos (Roldán \& Arelovich, 2020).

Una de las primeras tareas a realizar ha sido dar a conocer los objetivos del proceso participativo a los agentes locales dentro de dicho grupo de trabajo. Es muy importante explicar el concepto del Patrimonio Cultural Inmaterial; qué es, para qué sirve y su importancia como herramienta de identidad y cohesión social. Puede incluso plantearse la celebración de un pequeño taller de formación sobre PCI, aunque debe intentarse que no resulte demasiado teórico, y acercar el concepto de patrimonio inmaterial de una forma práctica y adaptada al colectivo al que se imparte. En el caso del proyecto en la Fontanalla no se ha celebrado un taller específico sobre patrimonio inmaterial, pero se ha trabajado y explicado el concepto en diferentes reuniones preparatorias. Cabe destacar que una buena parte de la comunidad local - vinculada al ámbito de las Artes y Oficios - ya estaba familiarizada con este concepto. También puede resultar útil la elaboración de materiales gráficos y escritos que expliquen de forma sencilla y directa el concepto de patrimonio inmaterial. Además, este material se puede utilizar para difundirlo a otros miembros de la comunidad, tal y como se ha hecho en el barrio de la Fontanalla (figura 1).

\section{Fases 2 y 3: propuesta técnica inicial de acciones metodológicas y definición y planificación de programa actuaciones por parte de la comunidad local}

Con el objetivo de que la propuesta inicial de acciones metodológicas del equipo de técnicos sea adoptada como propia por parte de la comunidad, resulta fundamental explicar con claridad los objetivos del proceso participativo y las acciones propuestas. A partir de la propuesta técnica, la comunidad local - representado por el grupo de trabajo - ha decidido llevar a cabo las acciones a), b), c) y d), si bien, hasta el momento, se ha ejecutado de forma completa la primera de ellas. Sólo desde el consenso y adaptación de 
estas acciones a las condiciones y realidad del barrio (definir qué acciones se van a realizar finalmente, cuándo se van a celebrar, dónde, quiénes van a asistir o presentar cada actividad, etcétera), la comunidad local puede adoptar el proceso y programa de actuaciones como algo propio.

\section{Figura 1 \\ Material de difusión empleado en proceso participativo}
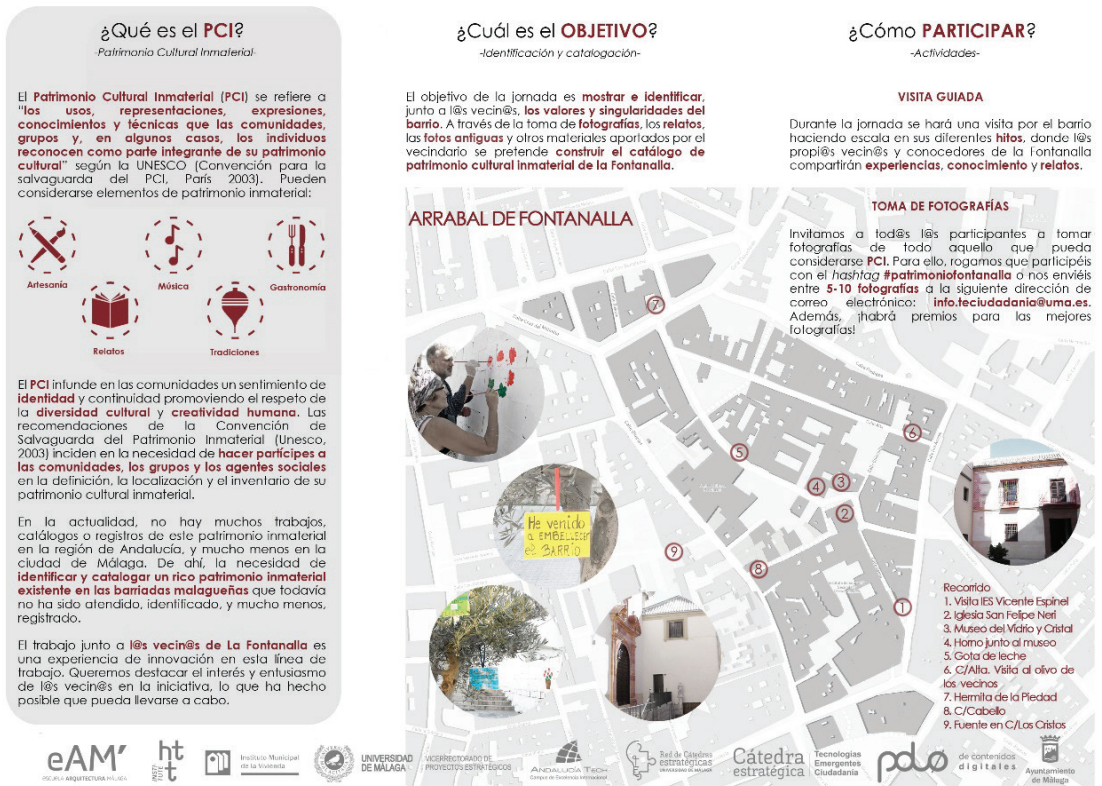

TOMA DE FOTOGRAFIAS

ARRABAL DEFONTANALA

Invitamos a todás 19 s participantos a tomor
fotografias de todo aquello que pueda fotografias de todo aquello que pueda
consideralse PCI. Para ello, logumos que porlicipés con el hashtag fpatimoniofontanalla o nos envies
entre 5 -10 fotografias a la siguiente dirección de correo electrónico: infoteciudadania@uma.es.
Ademós, ihabró premios para las mejores

\section{$+$}

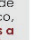

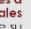
. de de
derial
davia

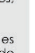
$=$ (9):
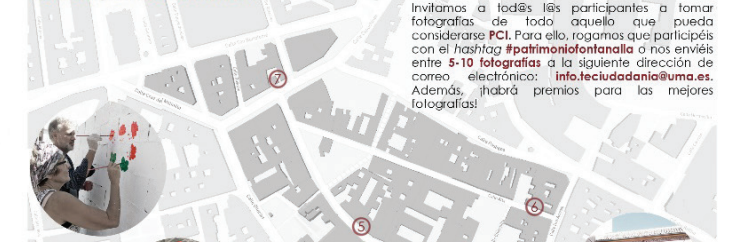

Fuente: Elaboración de Marta Córnax y Francisco J. Chamizo.

Cabe decir que los agentes intervinientes en el proceso de la Fontanalla han mostrado un gran interés y disponibilidad en el desarrollo de las diferentes actividades desde el primer momento. Se destaca la implicación de algunas personas del vecindario, todos ellos con un sentido fuerte de arraigo al barrio. Cada uno de los asistentes a las reuniones preparatorias ha mostrado interés por algunos de los aspectos planteados. El conocimiento de estos intereses personales, inquietudes y singularidades del barrio son los que deben terminar de conformar la metodología y proceso a seguir, transformando y precisando la propuesta técnica inicial de acciones a desarrollar. 
En el caso de la Fontanalla, se ha desarrollado el paseo colectivo guiado y se ha iniciado la acción metodológica de memoria del barrio o recopilación de fotografías. En las reuniones preparatorias del grupo de trabajo, se ha planteado, además, la posibilidad de elaborar un audiovisual que documente las costumbres y singularidades del barrio, y permita su difusión y el fortalecimiento de identidad cultural a través de los recursos de la etnografía audiovisual y comunicación social (Bruzón-Delgado, 2017). En este artículo se muestran los resultados de la acción 1 ya finalizada.

\section{Fase 3: ejecución de actividad programada: Paseo Colectivo Guiado}

Es importante que sean los agentes intervinientes quienes planifiquen, a su medida, cada una de las actividades programadas, acompañados y asesorados por especialistas. En el caso del paseo guiado por la Fontanalla, han sido ellos/as los que han definido los lugares a visitar, las personas que han presentado cada lugar, las historias y contenidos a relatar, etcétera. A partir de una planificación inicial, se han producido intercambios de experiencias y recuerdos de una forma espontánea que resultan de gran valor para construir la memoria colectiva. La actividad, a su vez, ha constado de diferentes fases: reuniones preparatorias de la visita guiada, la celebración de ésta y una jornada de valoración de la experiencia y recopilación de materiales resultantes.

En las reuniones preparatorias, los participantes han diseñado y decidido los elementos de interés que querían mostrar, incluyendo, dentro de estos elementos, algunas de las tradiciones, oficios artesanales como el del vidrio y la cerámica, relatos personales y leyendas del barrio, como la del "Monstruo de Calle Alta", recuerdos y homenaje a personas especiales por diferentes motivos, nuevas formas de socialización dentro del barrio, y lugares especiales por su capacidad para generar recuerdos de vivencias colectivas en ellos. Asimismo, se han incluido dentro de la visita, otros puntos de interés, como el instituto IES Vicente Espinel que, si bien puede vincularse a priori a un patrimonio arquitectónico tangible, representan lugares que generan apego y suscitan muchos recuerdos compartidos ligados a ellos. No obstante, cabría decir que una de las dificultades mayores encontradas en el proceso se relaciona, precisamente, con el concepto de patrimonio intangible: qué se puede considerar elemento patrimonial y su distinción entre tan- 
gible o intangible. Resulta muy necesario, establecer, desde un principio, unos criterios claros.

Estas reuniones preparatorias han resultado de gran interés por las discusiones y debates que en ellas se han producido acerca de los aspectos singulares y valores propios del barrio. Es importante documentar y registrar toda esta información a partir de notas, fotografías incluso vídeos. De no hacerlo, como en alguna de las reuniones celebradas, se pierde una información muy valiosa que debe ir construyéndose a lo largo del proceso. Es recomendable la elaboración de un documento abierto o listado de elementos singulares y que generan "apego" de una forma colectiva. En una fase posterior, se podrá valorar, cuáles de estos pueden considerarse elementos de patrimonio inmaterial de acuerdo a los requisitos generales de la Convención de París. El desarrollo inicial del proyecto ha permitido construir ese listado de elementos singulares si bien no se ha llegado a concluir cuáles van a formar parte de un catálogo de patrimonio inmaterial del barrio. La definición colectiva de esos elementos se determinará a lo largo de las diferentes actividades programadas dentro del proceso participativo.

Otro de los aspectos a destacar es la elección de guías y relatores de la visita. Este grupo de cicerones se ha formado con personas del vecindario del barrio y con otras personas y estudiosos que lo conocen en profundidad: historiadores, una arqueóloga y artesanos. Su conocimiento profundo del lugar ha resultado fundamental para definir y diseñar los hitos del paseo colectivo guiado. Resulta necesario, no únicamente definir esos hitos o paradas, sino hacer una planificación general de la visita: contenidos a mostrar, tiempo dedicado a cada lugar, etcétera. Asimismo, es recomendable la elaboración de material gráfico que describa la ruta, las etapas y el orden en que se van a realizar. De esta forma, los vecinos/as que no han podido asistir a la jornada completa, han podido hacerlo, al menos, a alguna de sus partes al conocer su ubicación y estimación en el tiempo. Se trata en definitiva de facilitar la participación en la experiencia al mayor número posible de personas.

Uno de los aspectos más interesantes de la experiencia se ha centrado en las aportaciones personales espontáneas de los/as vecino/as. De nuevo, se indica la necesidad de registrar todos los testimonios a través de vídeos, audios, o cualquier otro recurso disponible.

La actividad ha finalizado con la invitación a un refrigerio preparado por los organizadores (vecinos/as en su mayoría) tratándose de un momento lúdico en el que se ha producido el intercambio y acercamiento entre asisten- 
tes a la actividad. Estos espacios de participación resultan muy importantes para fortalecer las relaciones interpersonales dentro de la comunidad. Tras la celebración de la jornada, se ha llevado a cabo una reunión junto a agentes intervinientes para hacer una valoración de la experiencia, poner en común los materiales resultantes de ésta y empezar a planificar de forma colectiva la siguiente actividad.

Los resultados de la visita se consideran muy positivos desde el punto de vista de la asistencia, alcanzándose un número aproximado de 120 asistentes. La alta participación se traduce en una elevada repercusión de la experiencia, que ha permitido dar a conocer el barrio y sus singularidades más allá de los límites geográficos del mismo. Cabe decir que medios de comunicación locales se han interesado por el barrio y han dado a conocer la iniciativa (PTVMalaga, 2019). En términos de un análisis cualitativo, se hace una valoración desde diferentes puntos de vista. Los asistentes han sido, en gran parte, vecinos y vecinas del barrio, pero, también, han asistido algunos estudiantes, profesores de la universidad, técnicos de la administración local, incluso personas de otros barrios. Esta diversidad implica un interés por el barrio y su patrimonio por parte de la ciudadanía malagueña (y no únicamente de la comunidad local).

Resulta oportuno indicar que la participación en la actividad de algunos colectivos más desfavorecidos y recientes habitantes del barrio es baja en comparación con el grupo de personas que ha vivido durante muchos años en él. Se han contabilizado algunos asistentes de forma muy puntual. Con el objetivo de fomentar la cohesión social de la comunidad local, se plantea la necesidad de incorporar nuevos agentes sociales en el proceso participativo, tales como organizaciones ONG con sede en el barrio, y que se relacionan de forma activa y directa con estos colectivos. La incorporación de estos agentes sociales en las diferentes y futuras actividades planificadas pretende mejorar la gobernabilidad participativa del barrio, aumentar el número de relaciones interpersonales, así como incentivar el cuidado y respeto del entorno físico por parte de estos colectivos. Desde este punto de vista, se hace una valoración crítica de la metodología empleada. Resulta fundamental profundizar y conocer de manera exhaustiva la realidad y problemática social del barrio, incorporando agentes sociales locales en contacto con los colectivos más desfavorecidos desde un primer momento. En la actualidad, se trabaja de forma activa en la incorporación de dichos agentes al proceso. 
Por otro lado, y de cara a facilitar y fomentar la participación del mayor y diverso número de agentes, resulta importante desarrollar una estrategia de difusión de las actividades. En el caso de la Fontanalla, se valora de forma muy positiva la labor de difusión a través de las redes sociales, el "boca a boca" de los vecinos/as, la celebración de reuniones de vecinos donde se ha informado de las diferentes actividades. Asimismo, se considera que ha sido muy positiva la implicación de determinados agentes, como algunos representantes de la administración local, facilitando la difusión del evento en medios locales.

En la sesión de valoración de la actividad, surge de nuevo, en las discusiones, la falta de consenso y criterios comunes para discriminar entre patrimonio material e inmaterial, lo que denota la necesidad prioritaria de establecer unos criterios claros. Por otro lado, se hace una crítica a la falta de planificación en cuanto al tiempo dedicado a cada etapa, lo que ha provocado el retraso de las diferentes paradas y la imposibilidad de asistir a las últimas por parte de alguno/as asistentes. De la misma forma, hubiese sido deseable la realización de una encuesta de valoración de la experiencia a los participantes, incluyendo en ésta, posibles mejoras y sugerencias que pueden incorporarse en experiencias futuras similares. Se recomienda la incorporación de una encuesta de valoración de participantes en las siguientes actividades planificadas.

No obstante, y a pesar de la consideración de posibles mejoras en las fases sucesivas del proceso participativo, los primeros resultados obtenidos confirman la importancia de la participación y del desarrollo de acciones participativas para la salvaguardia del patrimonio inmaterial, y en concreto, para alcanzar la cohesión, la diversidad y creatividad en las comunidades locales. Los diferentes encuentros llevados a cabo - reuniones preparatorias, visita guiada, refrigerio organizado por vecinos/as y reunión de discusiónhan generado ricos debates que han puesto de manifiesto y valorizado algunos de las singularidades del barrio. Se han compartido historias e información a través de aportaciones espontáneas. Las experiencias más lúdicas han permitido el intercambio de recuerdos y vivencias que favorecen las relaciones interpersonales de la comunidad, y el sentido de pertenencia al barrio. Todas estas acciones han dado lugar a nuevas propuestas creativas como la futura organización de una exposición al aire libre en el barrio con todos los materiales e información recogida hasta el momento. 


\section{Conclusiones}

\section{Valoración de la metodología diseñada para la creación de espacios de participación en torno a la gestión del PCI}

El desarrollo metodológico se ha llevado a cabo, principalmente, a través de la creación de un grupo de trabajo local acompañado de técnicos/as, y la realización de talleres y reflexiones colectivas en torno al patrimonio inmaterial y singularidades del barrio. Se han planteado una serie de acciones metodológicas con carácter abierto y posteriormente, la comunidad local ha definido qué acciones llevar a cabo y ha planificado el proceso participativo. Tras la valoración de las primeras fases ejecutadas se hace una valoración muy positiva de la metodología y mecanismos empleados, en tanto que ha permitido la creación de espacios de participación entre vecinos/as de la comunidad local y con otros agentes externos al barrio. Los primeros pasos del proceso han permitido dar a conocer el barrio y su patrimonio inmaterial más allá de la comunidad local. La ejecución de las primeras fases apunta, sin embargo, la necesidad de incorporar nuevos agentes sociales relacionados con los colectivos más desfavorecidos del barrio, y con un menor índice de participación en el proceso.

Se hace una valoración positiva del proceso metodológico en cuanto a su flexibilidad y empoderamiento de los vecinos/as. Este empoderamiento se ha traducido en una alta implicación en las diferentes actividades realizadas. Una de las dificultades encontradas se relaciona con la gestión precisamente del patrimonio intangible: la falta de consenso y de criterios comunes a la hora de discriminar qué elementos pueden considerarse como tal. De todo ello se desprende la importancia de crear desde un inicio un grupo multidisciplinar con conocimientos, tanto en la gestión de procesos participativos, como en la gestión específica del PCI. Resulta esencial establecer unos criterios claros tanto en la fase de identificación, como en la de registro y catalogación de este tipo de patrimonio. Asimismo, se hace hincapié en la necesidad de documentar todo el desarrollo del proceso, no sólo las jornadas o hitos a priori más importantes, sino las reuniones preparatorias y otros encuentros donde se intercambian muchas experiencias y conocimiento de locales. 


\section{Patrimonio Cultural Intangible como elemento generador de procesos participativos}

El patrimonio cultural intangible de un lugar permite singularizar ese lugar, hacerlo diferente y especial, y generar entre sus habitantes, lazos o vínculos de identidad, hacia el lugar y entre sí. La búsqueda de valores y singularidades de forma general resulta un proceso muy enriquecedor para todas las personas, más si se hace en comunidad, permitiendo establecer lazos de identidad y cohesión.

Las diferentes reuniones preparatorias y jornadas realizadas son espacios de intercambio de información entre participantes, y al mismo tiempo, de convivencia y discusión, lo que, sin duda, ha permitido fortalecer las relaciones entre vecino/as con diferentes edades, campos profesionales, rangos sociales e inquietudes, pero todo/as ello/as con fuerte arraigo al barrio.

Asimismo, se ha dado a conocer algunas de las singularidades del barrio entre vecino/as y otros asistentes, con especial importancia de la actividad artesanal del vidrio y cerámica. Se ha dado a conocer la exposición del Museo del vidrío y se han mostrado las técnicas del oficio en uno de los talleres de artesanía, acercando así a toda la comunidad a una de las principales manifestaciones artísticas de la Fontanalla. Por otro lado, la experiencia ha fortalecido la relación del barrio con algunas instituciones implicadas como la universidad de Málaga, el Museo del Vidrio y Cristal, el centro IES Vicente Espinel, o el Ayuntamiento de Málaga.

El caso de la Fontanalla se suma, así, a otras experiencias participativas previas llevada a cabo que tienen por objeto dar a conocer y valorizar las singularidades y valores de su patrimonio cultural. Lerner (2005) nos recuerda que es necesario conocer para valorar, valorar para respetar, y respetar para amar al referirse a la importancia de fomentar el sentido de identidad hacia el lugar que habitamos. En este sentido, el patrimonio inmaterial se convierte en un motivo o herramienta activadora de procesos participativos que pretenden fortalecer la relación con el lugar y entre personas.

\section{Apoyos y soporte financiero de la investigación}

Entidad: Universidad de Málaga-Ayuntamiento de Málaga.

País: España

Ciudad: Málaga 
Proyecto subvencionado: (1) Cátedra Tecnologías Emergentes para la Ciudadanía / (2) Proyecto Málaga Net: bases para la rehabilitación sostenible del Patrimonio histórico de Málaga.

Código de proyecto: (1) 8.07/5.83.5026 / (2) 8.06/3.14.4955

\section{Agradecimientos}

Agradecemos su colaboración a los vecino/as de la Fontanalla que han participado en las actividades planificadas, a las instituciones y entidades implicadas: la asociación de vecinos/as y comerciantes de Arrabal Fontanalla, el Museo del Vidrio y Cristal de Málaga, el taller del vidrio Viarca, y el centro educativo I.E.S. Vicente Espinel. Agradecemos también su colaboración a los técnicos de investigación Marta Córnax Martín y Francisco J. Chamizo Nieto que han contribuido en la dinamización del proceso.

\section{Bibliografía}

Bruzón-Delgado, L. (2017). Audiovisual etnográfico y tradición: una contribución a la identidad y el desarrollo del municipio. La experiencia de San Juan Atitán (Guatemala). Universitas, XV(27), 45-65. http://bit.ly/3cZUw2S

Carofilis, N., \& García, G. (2015). El patrimonio como recurso: el cambio de paradigmas en la conservación urbana desde una perspectiva internacional. Estoa. Revista de La Facultad de Arquitectura y Urbanismo de la Universidad de Cuenca, 4(6) https://doi.org/10.18537/est.v004.n006.12

Carrera, G. (2009). Iniciativas para la salvaguardia del Patrimonio Inmaterial en el contexto de la Convención UNESCO, 2003: una propuesta desde Andalucía. Revista Patrimonio Cultural de España. http://bit.ly/2TPsIXq

Carrión-Gutiez, A. (2015). Plan Nacional para la Salvaguardia del Patrimonio Cultural Inmaterial. https://doi.org/10.4438/030-16-425-3

Craps, M., \& Brugnach, M. (2015). A relational approach to deal with ambiguity in multi-actor governance for sustainability. https://doi.org/10.2495/RAV150201

Dumas, N. Q. (2016). El futuro del pasado. Estoa. Revista de La Facultad de Arquitectura y Urbanismo de la Universidad de Cuenca, 4(6). https://doi. org/10.18537/est.v004.n006.04

Escalona-Hernández, C., Calvario-Morales, Á.F., García-Mora, M.T., Marín-Bolaños, B.A., Sonda de la Rosa, R., \& Valle-Cantón, O. (2017). Metodología 
para la identificación del patrimonio cultural inmaterial en la península de Yucatán. En Margarita de Abril Navarro Favela (Coord.), Estudios multidisciplinarios hacia el turismo sustentable en la Península de Yucatán (pp. 175-215). http://bit.ly/2TTt4N0

Frieri, S. (2014). Manual de herramientas participativas para la identificación, documentación y gestión de las manifestaciones del patrimonio cultural inmaterial. Bogotá. http://bit.ly/38T5B2G

Lacarrieu, M., \& Laborde, S. (2018). Diálogos con la colonialidad: los límites del patrimonio en contextos de subalternidad. Persona y Sociedad, 32(1), 11-38. http://bit.ly/2vjx5R8

Machuca-Santa Cruz, L. (1987). Málaga, ciudad abierta origen, cambio y permanencia de una estructura urbana. Colegio de Arquitectos. http://bit. ly/2QflBph

Mariano, M., Endere, M.L., \& Mariano, C.I. (2014). Herramientas metodológicas para la gestión del patrimonio intangible. El caso del municipio de Olavarría, Buenos Aires, Argentina. ICANH, Revista Colombiana de Antropología, 50(2), 243-269. https://doi.org/10.22380/2539472X53

Plan Nacional para la Salvaguardia del Patrimonio Cultural Inmaterial (2011). http://bit.ly/2Qf78cE Accesse 15/03/2020

PTVMalaga (2019). Tu barrio: Arrabal de Fontanalla. España: PTV Malaga. https:// bit.ly/30R1SC5

Racero-Calvo, J. (2014). Nuevos espacios culturales en la oferta turística de la ciudad de Málaga: El Museo del Vidrio y Cristal y su entorno. https:// bit.ly/33033nn

Roldán, D., \& Arelovich, L. (2020). Territorios en disputa. Los pescadores, la ribera, el urbanismo y el acuario del Paraná (Rosario, Argentina). Universitas, 32, 77-98. https://doi.org/10.17163/uni.n32.2020.04

Sánchez-Carretero, C., \& Jiménez-Esquinas, G. (2016). Relaciones entre actores patrimoniales: gobernanza patrimonial, modelos neoliberales y procesos participativos. Revista PH, 190. https://doi.org/10.33349/2016.0.3827

Sánchez-Gómez, J., Nebot Gómez de Salazar, N., \& García-Bujalance, N. (2013). Ensayos de reciclaje urbano como experiencia docente realizados por estudiantes de Arquitectura. Aprendiendo del barrio artesanal de La Funtanalla. En Aula Greencities (Ed.), Greencities y Sostenibilidad: Inteligencia Urbana aplicada a la Sostenibilidad Urbana (p. 15). Malaga. http://bit.ly/2U62Qpk 
UNESCO (2003). Convention for the Safeguarding of the Intangible Cultural Heritage. Paris, France. Retrieved from http://bit.ly/2IKh4qH

UNESCO Cataluña (2011). Metodología para el inventario del patrimonio cultural inmaterial en las REservas de la Biosfera. Barcelona. http://bit. ly/2vnjyrX

Van der Hammen-Malo, M.C., Frieri-Gilchrist, S., Sánchez-Silva, L.F., \& PeñaBautista, A. (2015). Opciones y Acciones para la salvaguardia del PCI: una compilación de experiencias (Bogotá: Mi). Bogotá. Retrieved from http://bit.ly/38RQLcP Accessed 15/03/2020

Fecha de envío: 2020/03/19; Fecha de aceptación: 2020/08/10;

Fecha de publicación: 2020/09/01 